\title{
Re-Engineering Enterprise Architectures
}

\author{
Murat Paşa Uysal \\ Baskent University, Baglica \\ Kampusu, 06790, Ankara, Turkey \\ Email: muysal@baskent.edu.tr
}

\author{
Ali Halıcı \\ Baskent University, Baglica \\ Kampusu, 06790, Ankara, Turkey \\ Email: ahalici@baskent.edu.tr
}

\author{
A. Erhan Mergen \\ Rochester Institute of Technology, \\ Rochester, NY, USA \\ Email: emergen@saunders.rit.edu
}

\begin{abstract}
An Enterprise Architecture (EA) is used for the design and realization of the business processes, along with user roles, applications, data, and technical infrastructures. Over time, maintaining an EA update may become a complex issue, let alone an organization-wide architecture and its related artifacts. EA practices provide much of the required guidelines for the design and development of EAs. However, they cannot present a comprehensive method or solution for the re-engineering processes of EAs. In this paper, we propose an EA re-engineering model and present its potential contributions. The study is conducted according to the Design Science Research Method. The research contribution is classified as an "application of a new solution (process model) to a known problem (re-engineering EA)". The future research efforts will focus on the implementation and evaluation of the model in case studies for gathering empirical evidences.
\end{abstract}

\section{INTRODUCTION}

$\mathrm{T}$ HE volume of Information Technologies (IT) has been growing more than expected and the dependency on IT continues to increase as well. The industry has witnessed the development of information systems, and a great majority of them has been built over the past decades. Today, it is nearly impossible to think of an enterprise without IT and also its applications which are based on various business processes and infrastructure. One instance is an Enterprise Architecture (EA); it is defined as a "coherent whole of principles, methods, and models that are used in the design and realization of an enterprise's organizational structure, business processes, information systems, and infrastructure [1]". Being a discipline representing an enterprise in various aspects, it is also a means to facilitate communication between different types of stakeholders in an organization.

There have been various frameworks for EAs, such as TOGAF, Zachman, DoDAF, etc. [2]. While presenting the core concepts, definitions and a basis for EAs, they also provide methods and techniques for the design and development of EAs. Thus, EA models can be used by different stakeholders in an organization to support the decision-making processes. A large organization or an enterprise can consist of different architectural elements, such as processes, applications, and technical infrastructures. For example, Figure 1 represents a small to medium size enterprise with a generic EA model:

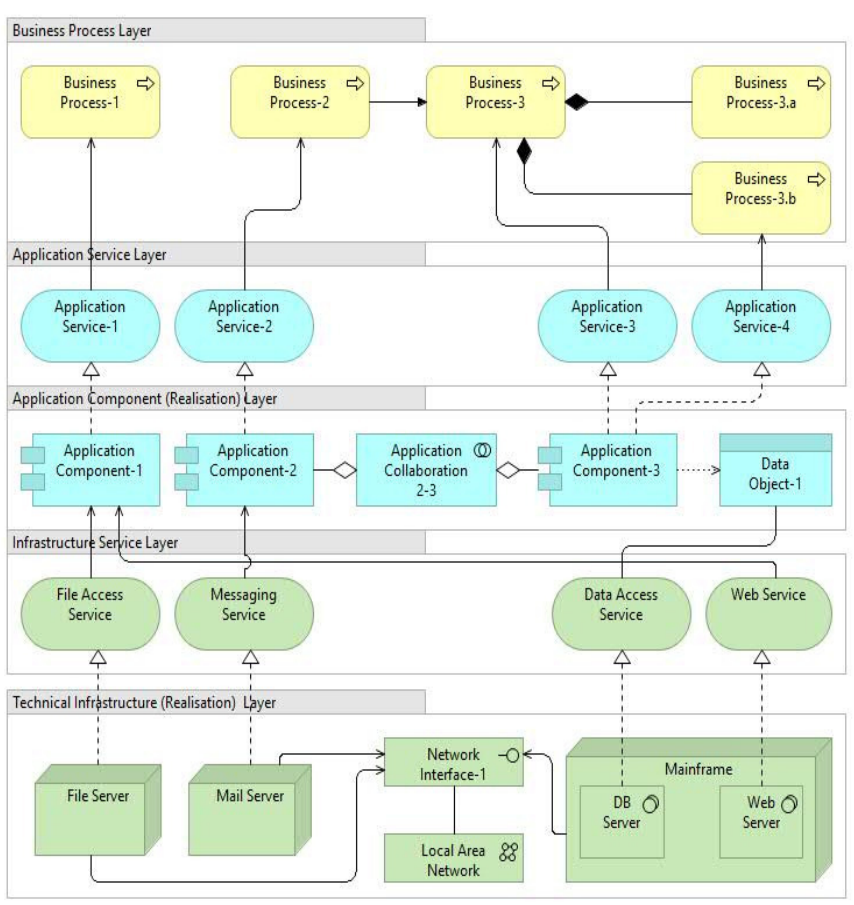

Fig.1. Enterprise Architecture

Only are the business, application and technical layers, each with simple components, included, and we exclude the layer for users and roles for simplicity purpose. In recent years, changes in various aspects of business and technology have also made some changes to the design, development or maintenance of EAs inevitable. In this context, the everchanging organizational environment necessitates the architects take required measures to reflect these changes to the EAs.

A great body of knowledge has been accumulated [3] in EA and thus, EA practices can provide much of the required guidelines, methods and techniques for the development or management of EAs. Implementing a change and assessing its impact are the two important activities when improving EAs. Over time, keeping or maintaining a single IT system and component update becomes a complex issue [4], let alone managing a large organizational architecture and its 
related IT artifacts. On the other hand, developing an EA from scratch is usually cumbersome and resourceconsuming process; in addition, discarding or re-developing encompasses many risks as well. Therefore, poor maintenance of EAs may require the re-engineering of EAs. Re-engineering can be defined as "the examination or alteration of a subject system to reconstitute it in a new form and subsequent implementation of that form [5]". It improves the understanding of a system and its structure for increased maintainability, reusability, and evolvement. Although re-engineering has potential for contributing to the EA knowledge domain, the review of literature on EA cannot provide sufficient examples and indicates that it is still a less-explored research topic [3].

In this study, therefore, we propose an EA re-engineering process model and present its potential contributions. This model, along with its prescriptions for improving EA reengineering, can be regarded as the main contributions of our work. The other parts of this paper include the method, proposed model, and conclusion sections respectively.

\section{METHOD}

We followed the guidelines of Design Science Research (DSR) for this study [6]. This research method focuses on the creation of scientific knowledge when solving a realworld problem and developing IT artifacts in the Information Systems (IS) domain [7]. The research output is the "application of a new solution (process model) to a known problem (re-engineering EA) [8]". problem. Therefore, the acceptance criteria for the evaluation of the proposed model were defined as follows:

- The model was expected to consider and reflect the concerns of stakeholders relevant to EAs during the reengineering process.

- It should also allow the use and integration of tools, techniques, and experiences that may belong to other knowledge bases, such as software engineering and information systems.

The model was developed during the design-buildevaluate phase, at which the critical research activities were conducted. This was also an iterative and incremental process with the generation of design alternatives [7]. However, the evaluation of the EA re-engineering model was left to the next paper because of research limitations.

\section{RE-ENGINEERING AN ENTERPRISE ARCHITECTURE}

\section{A. Requirement Analysis and Specification}

Our proposed process model is given in Figure 2. EA reengineering starts with determining the current state of the architecture and specifying new structural, behavioral, and quality requirements which reflect the views of all stakeholders. Actually, the requirements management is a continuous process that ensures any changes to the requirements are handled and reflected in other phases. One important point here is scoping the architectural activities according to the re-engineering objectives, stakeholder concerns, availability of people and resources. Moreover,

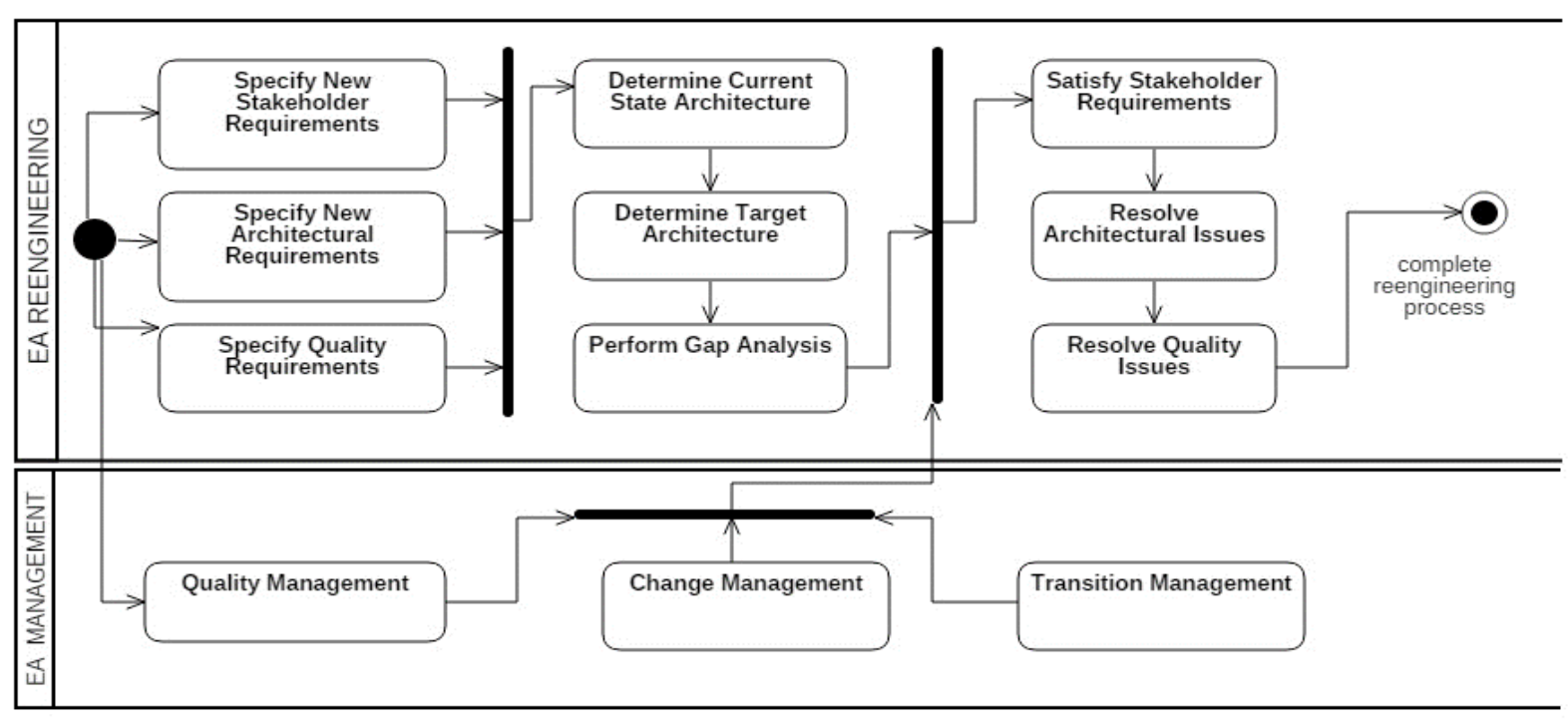

Fig. 2. EA Re-engineering Process Model

The study began with identifying a problem in the EA application environment. This was the need for a model that would guide the EA re-engineering processes. Current EA practices could provide the knowledge for the design, development and evaluation of EAs. However, how to reengineer EAs is missing in the research. The other important issue was the solution specification for this research these dimensions should be considered as well: (a) breadth (the part that re-engineering efforts will deal with); (b) depth (the level of detail that the re-engineering efforts will go); (c) time period; and (d) any or all of the architecture domains (business, data, application, technology) [9].

One issue is to know how an organization is capable of conducting the EA design and development processes, as well as the re-engineering. Capability Maturity Models 
(CMMs) can address this problem by providing effective and proven methods and practices. It has already been indicated that a successful EA practice needs to establish its capabilities in the management areas, such as financial, performance, service, resource, risk, stakeholder, configuration [9].

Another issue during the re-engineering of an EA is to specify the requirements for the quality of both current and target EAs. Several works propose different types of quality criteria for EAs; however, the literature review cannot provide a comprehensive framework or complete solution [10]. Although it is dedicated to software product quality only, we believe that ISO Quality Requirements and Evaluation (SQuaRE) standard [11] has great potential for providing the benefits of defining, measuring, and evaluating the quality of EAs for both an artifact and a reengineering process. Therefore, we adopt the SQuaRE framework, which is the approved series of standards for software quality. It has five divisions that cover modeling, managing, specifying, measuring, and evaluation of the quality of various software products. Its main purpose is to guide and assist people who acquire or develop software products with the quality requirements.

SQuaRE considers three areas as vital for assuring quality [12]. The (a) "internal quality" which is the degree to which static attributes of a product satisfy the stated needs. The (b) "external quality" indicates the behavior of how the system satisfies the needs in a testing environment. Finally, (c) the "quality in use" determines whether a product meets the requirements of the specified users in a realistic environment. In this framework, thus, the quality in use depends on the external quality, and the external quality depends on the internal quality respectively. This standard also provides different sets of quality measures, most of which can be used for EAs. Functional suitability, efficiency, compatibility, usability, reliability, maintainability are some of the measures. Therefore, the quality management process of an EA in Figure 2 can be accomplished by the SQuaRE standards. After the requirement analysis and specification, the following main steps are taken during the EA re-engineering process:

\section{B. Determine the Scope, Breadth and Depth of EA:}

In most cases, requirement analysis and specifications will naturally drive the whole process. However, determining the scope and breadth of the re-engineering project is still the first and important challenge. Although a complete EA is expected to address all of the business, data, application, and technology architecture domains [9], a single, all-inclusive, organization-wide architecture may be too complex and resource consuming. In this case, focusing on particular business segments, specific organizational and quality requirements may be suggested. For some cases, creating the EA as a federation of architectures may be another option, although it would bring additional issues, such as consistency, maintenance, and integration. Also, special care should be given to the depth of the EA, which indicates the appropriate level of detail to be captured during re-engineering. This level can be relevant to the extent that the required details are included while the unnecessary ones are excluded for the sake of usefulness and simplicity.

\section{Determine Current State, Reference and Baseline Architectures:}

An EA re-engineering project usually results from the deficiencies of current $\mathrm{EA}(\mathrm{s})$ or new enterprise requirements. However, the re-use of appropriate architectures existed in the repository (if exist) may be suggested. Reference architectures and architectural patterns accepted within the organization, or previous architectural work relevant to the project outcomes may be used for the baseline architecture descriptions.

\section{Determine Target Architectures:}

The target architecture(s) represents overall and final descriptions of a future state of the EA or its part that is being re-engineered. They demonstrate a response to the project goals and the concerns for the functional and quality requirements of the current EA. To this end, architects can also make use of foundation and common systems architectures [9], as well as the organization-specific architectures or elements that may be re-usable when considering the re-engineering objectives. While a foundation architecture consists of principles and generic components, a common system architecture may be a network or operation architecture, but it is still incomplete in terms of general system functionality [9]. At a more detailed or specific level, industry architectures, which reflect the standards and requirements specific to an industry, can be utilized for the specification of target EA(s).

\section{E. Perform Gap Analysis and Determine Transition Architectures:}

After determining the target architectures, we conduct a gap analysis to identify the differences between the states of current and target EAs. Transition Architectures are evolutionary in nature and converge on the targets while describing the specifics of each increment in line with the architecture descriptions. If the project requires a large scale architectural transformation, it is advisable to address the issues of the EA layers to the extent that the re-engineering objectives are met. For example, much of the focus may be given to an Enterprise Information System Architecture, which is composed of two main domains: data and application architectures [9].

Data architecture domain includes data entities used by business processes, functions, or services, as well as showing how data are created, stored, reported and transported. It is also important to note the level of data complexity, data migration, and data integration requirements needed to support data exchanges between applications. Therefore, architecture definition documents comprise some or all of the business, logical and physical data models along with the diagrams, such as conceptual, logical, security, and migration. 
The other domain is Application Architecture that enables business architecture and addresses the stakeholder concerns. While developing this type of architecture, an architect can consider the following: (a) forming a list of applications as a portfolio; (b) decomposing complicated applications into simplified applications; and (c) using different matrices to relate the application architecture to corresponding business and data architectures. How the applications will function and handle the integration, migration, and operational concerns is another issue. For this purpose, TOGAF proposes the use of diagrams, such as application, use-case, realization, and migration, for this process [9].

\section{F. Quality Management:}

Another important issue is defining the quality attributes of the target EAs. As mentioned before, the SQuaRE standards provide the required guidance for reflecting on the stated and implied quality needs. The focus, therefore, should be on what to measure, how to conduct measurements, and how to evaluate the EA's characteristics influencing its quality. For example, it is possible to use the ISO/IEC 2502n Quality Measurement Standards and their external and quality-in-use measures for the EA elements belonging to Application and Data Layer [13] though it may not be practically possible to include all scenarios and still conduct the quality assurance procedures. Considering the fact that different quality measures are dependent on the EA re-engineering goals, the ISO/IEC 2501n quality models [12] can be tailored. The enterprise resources required for the quality management should also be allocated according to the project objectives.

\section{G. Change and Transition Management While Resolving Architectural Issues}

We develop an implementation and migration plan, as well as an architecture road map to take into account the gaps between the baseline and target architectures [9]. The migration plan is a mean to move from the baseline and transition architectures to the target architectures. The changes are logically grouped into work packages in transition architectures, and the project team concentrates on how to improve the EA. The proposed solutions usually indicate the construction and specification of the architectures at the corresponding levels of the target EA. While trying to ensure conformance with the target architecture, the other re-engineering activities would be the assessment of dependencies between EA elements, costs and benefits, estimating the time and resource requirements and so on. Consequently, the target architectures are deployed and delivered as a series of EA transitions. This also enables early realization of the expected business benefits, reflecting on the business priorities, and thus, minimizing the possible risks during the implementation of an EA re-engineering program.

\section{CONCLUSION}

As the volume of IT and the dependency on IT increases, so does the variety of IT management methods and tools. An EA is an example of having a lot to do with enterprise IT management. It, therefore, has been gaining popularity in the IS research community. The current EA practices may provide much of the required guidelines for the design and development of EAs; however, they are still far away from presenting a comprehensive solution to the problems of EA re-engineering.

In this paper, we proposed an EA re-engineering process model and outlined its main steps and components. Additionally, we adopted a quality management framework, not only for the re-engineering purposes, but also for the whole EA design and development processes. However, the research and space limitations led us to present only the conceptual background. Therefore, our future research efforts will focus on the implementation and evaluation of the proposed model in case studies for gathering empirical evidences.

\section{REFERENCES}

[1] M. Lankhorst, "Enterprise Architecture at work: Modelling, communication, and analysis", Springer-Verlag Berlin Heidelberg, 2009 .

[2] EABOK, Guide to the (Evolving) Enterprise Architecture Body of Knowledge, the MITRE Corporation, 2004.

[3] B.D. Rouhani, M.N. Mahrin, F. Nikpay, R.B. A.P. Nikfard, "A systematic literature review on enterprise architecture implementation methodologies", Information and Software Technology, vol.62, pp. 120, 2015.

[4] H.K Dam, Lam-Son Le, A. Ghose, "Managing changes in the enterprise architecture modelling context", Enterprise Information Systems, vol. 10:6, 666-696, DOI: 10.1080/17517575.2014.986219, 2016.

[5] E. Chikofsky, J. Cross, "Reverse engineering and design recovery: A taxonomy", IEEE Software, vol. 7, 1, pp.13-18, 1990.

[6] A. Hevner, S. Chatterjee, "Design Research in information systems", Integrated Series in Information Systems, vol. 22, DOI 10.1007/978-1, 2010.

[7] V.K. Vaishnavi, W.J. Kuechler, Design Science Research methods and patterns: Innovating information and communication technology, USA, Auerbach Publications, Taylor \& Francis Group, 2008.

[8] S. Gregor, A.R. Hevner, "Positioning and presenting design science research for maximum impact", MIS Quarterly, vol.37, 2, pp.337-355, 2013.

[9] Open-Group, TOGAF Version 9.1 Evaluation Form, published in the U.S. by The Open Group, 2011.

[10] SEI, A workshop on analysis and evaluation of enterprise architectures, CM Software Engineering Institute, 2010.

[11] SQuaRE, Quality management division, ISO/IEC 2500n, 2014.

[12] SQuaRE, Quality model division, ISO/IEC 2501n, 2014.

[13] SQuaRE, Quality measurement division, ISO/IEC 2502n, 2014. 